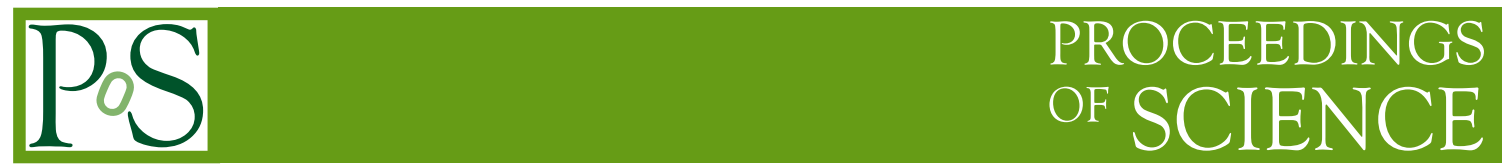

\title{
Searches for new physics at HERA
}

\author{
Hayk PIRUMOV*广 \\ PI Heidelberg \\ E-mail: hayk.pirumov@desy.de
}

The latest results on a variety of searches for new physics at HERA by the H1 Collaboration are presented. HERA, the world's only ep Collider at DESY, Hamburg, running $1991-2007$ at center-of-mass energies up to $320 \mathrm{GeV}$, was ideally suited for searches for physics beyond the Standard Model due to its unique initial state. The searches use the complete HERA data sample of the $\mathrm{H} 1$ experiment corresponding to a total integrated luminosity of about $0.5 \mathrm{fb}^{-1}$. Topics covered include a search for eqeq contact interactions, a search for first generation leptoquarks, a search for lepton flavour violation, and a search for squark production in R-parity violating supersymmetry models.

The 2011 Europhysics Conference on High Energy Physics-HEP 2011,

July 21-27, 2011

Grenoble, Rhône-Alpes France

${ }^{*}$ Speaker.

${ }^{\dagger}$ On behalf of the H1 collaboration. 


\section{Search for Contact Interactions}

New physics processes with characteristic mass scales in the $\mathrm{TeV}$ range may be present in deep inelastic $e^{ \pm} p$ neutral current (NC) scattering at very high $Q^{2}$. The four fermion contact interaction (CI) concept may be exploited as a method to investigate the interference between any new particle field with the Standard Model boson field.

A search for deviations of the measured neutral current cross sections from the SM prediction at high $Q^{2}$ is performed by the $\mathrm{H} 1$ collaboration [1]. The analysis is based on the full $\mathrm{H} 1 \mathrm{NC}$ data that corresponds to an integrated luminosity of $446 \mathrm{pb}^{-1}$. Since no significant deviations are observed, various BSM models can be constrained. Limits at $95 \%$ confidence level are derived on the effective mass scale $L$ in eqeq contact interactions $(L>3.6-7.2 \mathrm{TeV})$, on the mass to the Yukawa coupling ratio for leptoquark(LQ) models $\left(M_{L Q} / \lambda_{L Q}>0.41-1.86 \mathrm{TeV}\right)$, on the effective Planckmass scale in models with large extra dimensions $\left(M_{S}>0.90 \mathrm{TeV}\right)$ and on the quark electroweak charge distribution radius $\left(R_{q}<0.65 \cdot 10^{-18} \mathrm{~m}\right)$.

\section{Search for First Generation Leptoquarks}

Neutral and charged current data at high $Q^{2}$ are also used in a dedicated search by the $\mathrm{H} 1$ experiment for first generation scalar and vector leptoquarks produced in $e p$ collisions at HERA [2]. The full $\mathrm{H} 1$ data sample is used in the analysis, corresponding to an integrated luminosity of $446 \mathrm{pb}^{-1}$. No evidence for the production of leptoquarks is observed in final states with a large transverse momentum electron or with large missing transverse momentum and constraints on leptoquark models are derived.

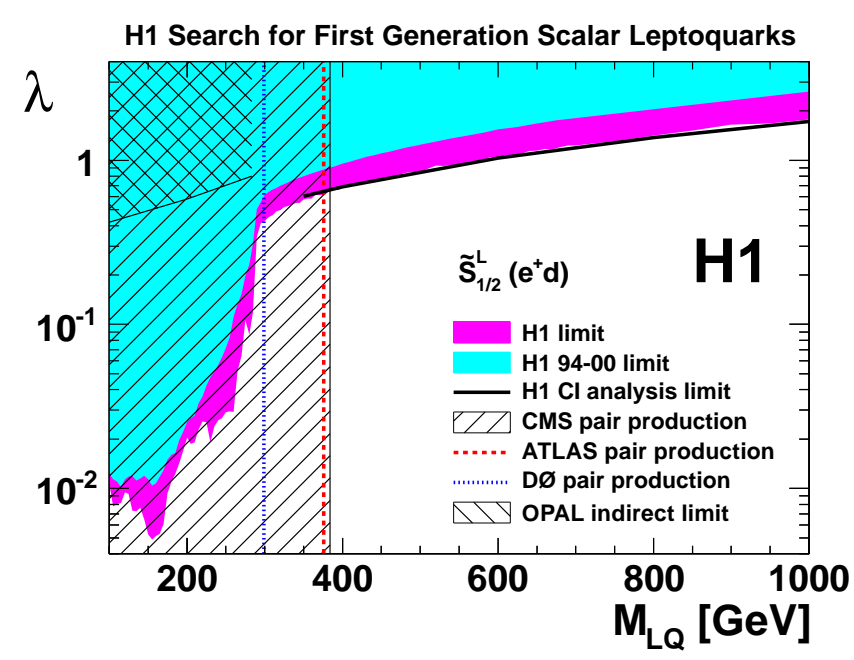

Figure 1: Exclusion limits on the coupling $\lambda$ as a function of the leptoquark mass for the $\tilde{S}_{1 / 2}$ in the framework of the BRW model. The parentheses after the LQ name indicate the fermion pairs coupling to the LQ, where pairs involving anti-quarks are not shown. Domains above the curves are excluded at $95 \%$ CL. Indirect limits from LEP (OPAL), the Tevatron (D0) and the LHC (CMS and Atlas $\sqrt{s}=7$ TeV data) are shown for comparison, as well as constraints on LQs with masses above $350 \mathrm{GeV}$ from the $\mathrm{H} 1$ contact interaction analysis. Limits from the previous $\mathrm{H} 1$ publication are also included. 
An example of the exclusion limits on the coupling $\lambda$ as a function of the $\tilde{S}_{1 / 2}$ leptoquark mass is shown in figure 1. The new H1 limits extend beyond the reach in LQ mass excluded by previous searches at HERA. For leptoquark couplings of electromagnetic strength $\lambda=0.3$, first generation leptoquarks with masses up to $800 \mathrm{GeV}$ are excluded at $95 \%$ confidence level.

\section{Search for Lepton Flavour Violation}

A search for the lepton flavour violating processes $e p \rightarrow \mu X$ and $e p \rightarrow \tau X$, mediated by second and third generation leptoquarks, is performed by H1 [3] using a data sample corresponding to an integrated luminosity of $0.41 \mathrm{fb}^{-1}$. No evidence for lepton flavour violation is found in final states with a muon or tau-lepton and a hadronic jet. Limits are derived on the mass and the couplings of leptoquarks inducing lepton flavour violation in an extension of the Buchmüller-Rückl-Wyler (BRW) framework.

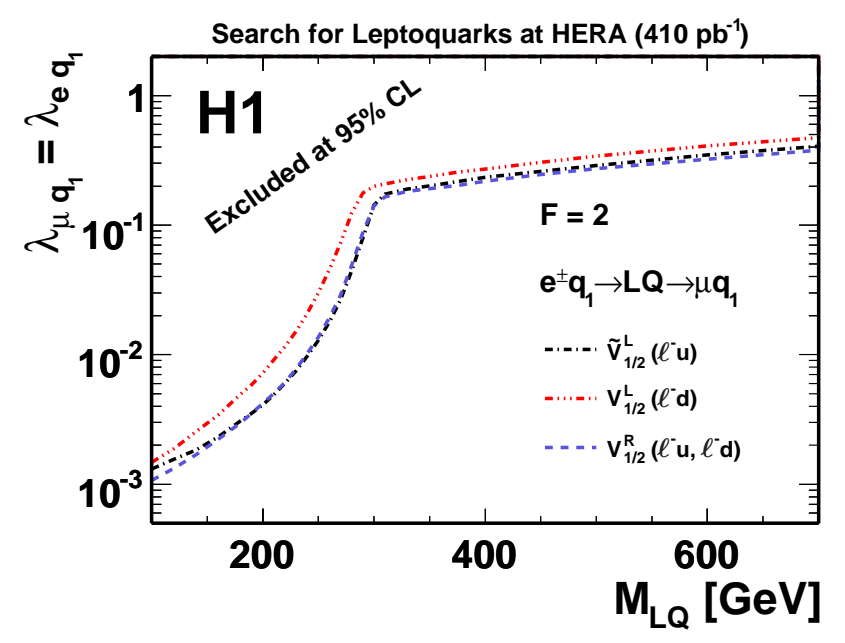

Figure 2: Exclusion limits on the coupling constants $\lambda_{\mu q}=\lambda_{e q}$ as a function of the leptoquark mass $M_{L Q}$ for vector $L Q s$ with $F=2$. Regions above the lines are excluded at $95 \% \mathrm{CL}$. The notation $\mathrm{q} 1$ indicates that only processes involving first generation quarks are considered. The parentheses after the LQ name indicate the fermion pairs coupling to the LQ, where pairs involving anti-quarks are not shown.

Exclusion limits for three different vector-type LQs are shown in figure 2. For a coupling $\lambda$ of electromagnetic strength leptoquarks with masses up to $712 \mathrm{GeV}$ and $489 \mathrm{GeV}$ in the muon and tau channels, respectively, can be excluded at $95 \%$ confidence level.

\section{Search for Squark Production at HERA}

A search for squarks in R-parity violating supersymmetry is performed [4]. The resonant production of squarks via a Yukawa-type coupling $\lambda^{\prime}$ is considered, taking into account all relevant direct and indirect R-parity violating decay modes. No evidence for squark production is found in the framework of the Minimal Supersymmetric Standard Model(MSSM) and in the Minimal Supergravity Model(mSUGRA). The results are interpreted in terms of exclusion limits on the masses of squarks. The parameter scan in the MSSM parameter space is performed (figure 3). Squarks 
of the first and second generation with masses up to $275 \mathrm{GeV}$ are excluded in the considered part of the parameter space for a Yukawa-type coupling of electromagnetic strength at $95 \%$ confidence level.
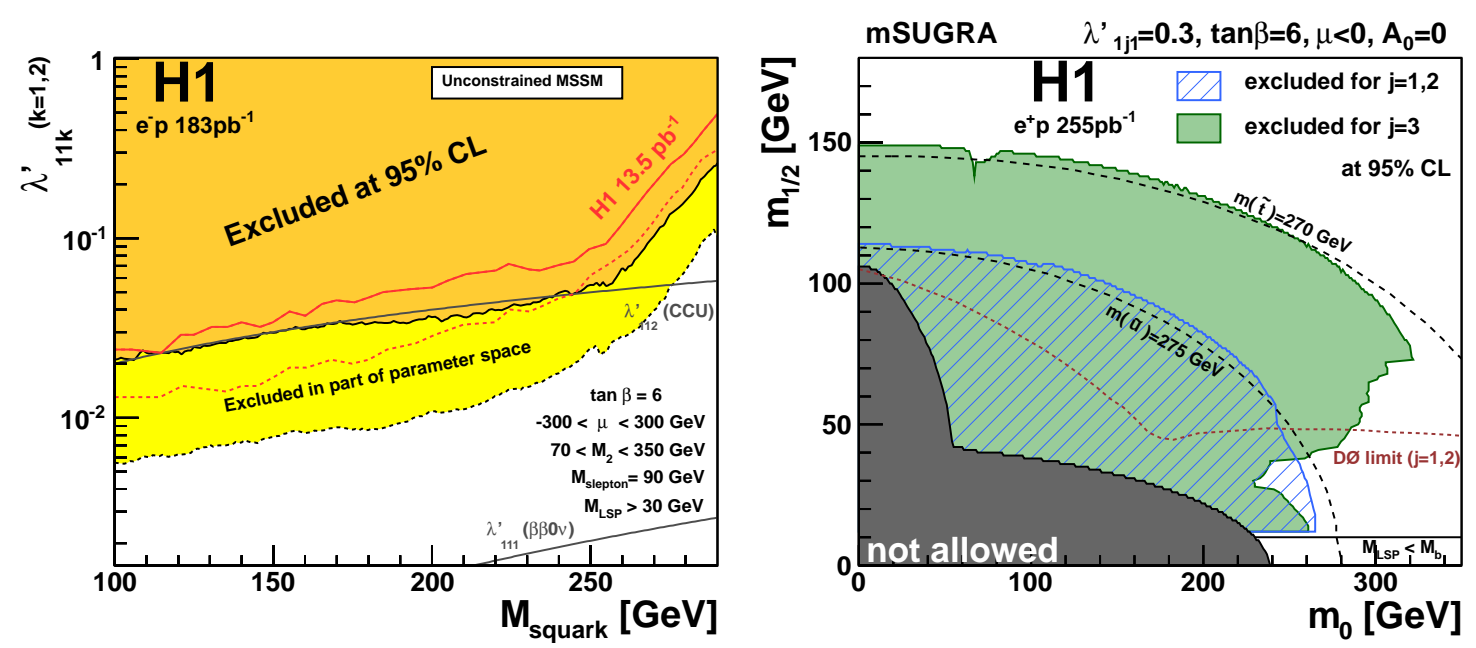

Figure 3: On the left the exclusion limits on $\lambda_{11 k}^{\prime}$ for $\mathrm{k}=1,2$ as a function of the squark mass from a scan of the MSSM parameter space are presented. The dark filled region indicates values of the coupling $\lambda_{11 k}^{\prime}$ excluded in all investigated scenarios whereas the light filled region is excluded only in part of the scenarios. Indirect limits from neutrinoless double beta decay experiments and tests of charged current universality are also shown. For comparison, the corresponding limits from the previous $\mathrm{H} 1$ analysis are also indicated. On the right the exclusion limits in the $m_{0} ; m_{1 / 2}$ plane assuming $\lambda_{1 j 1}^{\prime}=0.3$ for $\tan \beta=6$ for $j=1,2$ (hatched region) and $j=3$ (light filled region) are shown. Curves of constant squark mass are illustrated for $m(\bar{u})=275 \mathrm{GeV}$ and $m(\bar{t})=270 \mathrm{GeV}$. Also indicated are constraints obtained by the D0 experiment at the Tevatron. The dark filled region labeled as "not allowed" indicates where no REWSB solution is possible or where the LSP is a sfermion.

\section{Summary}

The complete HERA data sample of the H1 experiment with a total integrated luminosity of about $0.5 \mathrm{fb}^{-1}$ is analyzed in searches for new physics. The data show good agreement with the SM predictions. Limits on contact interactions, leptoquarks and R-parity violating supersymmetry are derived.

\section{References}

[1] F. D. Aaron et al. [H1Collaboration], Phys. Lett. B 705, 52 (2011) [arXiv:1107.2478].

[2] F. D. Aaron et al. [H1Collaboration], Phys. Lett. B 704, 388 (2011) [arXiv:1107.3716].

[3] F. D. Aaron et al. [H1Collaboration], Eur. Phys. J. C 71, 1572 (2011) [arXiv:1011.6359].

[4] F. D. Aaron et al. [H1Collaboration], Eur. Phys. J. C 71, 1572 (2011) [arXiv:1011.6359]. 